\title{
GROUPS WITH TRIVIAL SCHUR MULTIPLICATOR
}

Dedicated to the memory of Hanna Neumann

\author{
J. W. WAMSLEY
}

(Received 21 June 1972, revised 13 March 1973)

Communicated by M. F. Newman

\section{Introduction}

Let $G$ be a finite group generated by $n$ elements and defined by $m$ relators, then $G$ has a presentation

$$
G=\left\{x_{1}, \cdots, x_{n} \mid R_{1}, \cdots, R_{m}\right\}=F / R,
$$

where $F$ free on generators $x_{1}, \cdots, x_{n}$ and $R$ is the normal closure in $F$ of $R_{1}, \cdots, R_{m}$. The deficiency of this presentation is $n-m$ and the deficiency of $G$ is the maximum over all presentations for $G$.

The torsion part of $R /[F, R]$ is a presentation invariant and is known as the Schur multiplicator of $G$. The question as to how the number of generators of the Schur multiplicator is related to the deficiency is as yet unanswered. Neumann [3] asked if every finite group with trivial multiplicator has a presentation with equal numbers of generators and relators. Swan [4] exhibited a family of soluble groups with trivial multiplicator and unbounded deficiency. The question however is still unanswered for finite nilpotent groups and in this paper we answer the question in the affirmative for groups of order $3^{n}, n \leqq 6$, as classified by James [1].

\section{Preliminaries}

In this section we develop some results concerning the deficiency of factor groups of groups with zero deficiency. The following theorem (Theorem 2.1 [5]) is stated without proof,

THEOREM 2.1. Let $G$ be a finite p-group with presentation $G=F / R$ with $F$ free on $x_{1}, \cdots, x_{n}$ and suppose the vector space $R /[F, R] R^{p}$ has dimension $m$. If we take any set of $m$ elements $R_{1}, \cdots, R_{m}$, of $R$, linearly independent in $R$ modulo $[F, R] R^{p}$ and let $K=F / S$ where $S$ is the normal closure of $R_{1}, \cdots, R_{m}$ in $F$, then 
$G$ is the maximal p-factor group of $K$, in the sense that if $A$ is a finite p-group which is a factor group of $K$ then $A$ is a factor group of $G$.

COROLlaRY 2.2. Let $M=\left\{x_{1}, \cdots, x_{n} \mid R_{i_{1}}, \cdots, R_{i_{1}}\right\}$ where $R_{i_{1}}, \cdots, R_{i_{t}}$ is a subset of $R_{1}, \cdots, R_{m}$. If $M$ is a finite p-group then $G=K$.

Proof. $K$ is a factor group of $M$ and hence $K$ is a finite $p$-group.\|

LeMma 2.3. Let

and

$$
G=\left\{x_{1}, \cdots, x_{n} \mid R_{1}, \cdots, R_{m}\right\}=F / R
$$

$$
G / N=\left\{x_{1}, \cdots, x_{n} \mid R_{1}, \cdots, R_{m}, S_{l}, \cdots, S_{t}\right\}=F / S
$$

then if $R_{i_{1}}, \cdots, R_{i}{ }^{\mu}$ are linearly independent in $S$ modulo $[F, S] S^{p}$ they are linearly independent in $R$ modulo $[F, R] R^{p}$.

Proof. The natural mapping $R /[F, R] R^{p}$ into $S /[F, S] S^{p}$ is a homomorphism and hence a linear transformation of the respective vector spaces.\|

THEOREM 2.4. Let $G$ be a finite p-group with zero deficiency and $N$ normal subgroup of $G$ contained in the derived group of $G$, then $G / N$ has trivial multiplicator if and only if $N=1$.

Proof. $G, G / N$ and $G /[G, G]$ have presentations

$$
\begin{aligned}
G & =\left\{x_{1}, \cdots, x_{n} \mid R_{1}, \cdots, R_{n}\right\}=F / R, \\
G / N & =\left\{x_{1}, \cdots, x_{n} \mid R_{1}, \cdots, R_{n}, S_{1}, \cdots, S_{t}\right\}=F / S, \text { with } S_{i} \text { in }[F, F]
\end{aligned}
$$

and

$$
G /[G, G]=\left\{x_{1}, \cdots, x_{n} \mid R_{1}, \cdots, R_{n}, S_{1}, \cdots, S_{t}, x_{i} x_{j} x_{i}^{-1} x_{j}^{-1}\right\}=F / T
$$

respectively.

Now $R_{1} \cdots, R_{n}$ are linearly independent in $T$ modulo $[F, T] T^{r}$ and hence by lemma 2.3 are linearly independent in modulo $[F, S] S^{p}$. If $G / N$ has trivial multiplicator then the dimension of $S /[F, S] S^{p}$ is $n$ whence, by theorem $2.1, G / N$ is the maximal $p$-factor of $G$. However $G$ is a finite $p$-group and threefore $G / N=G$, i.e. $N=1$.

We now give an application of these results to specific p-groups considered by Macdonald [2] with

THEOREM 2.5. Let $G$ be the finite p-group, $p \geqq 3$, with presentation

$$
\begin{array}{r}
G_{1}=\left\{a, b, c \mid c=a^{-1} b^{-1} a b, c^{-1} a c=a^{1-p^{\alpha}}, c b c^{-1}=b^{1+p^{\beta}}, a^{p^{\alpha+\beta}}=b^{p^{2 \beta}}=c^{p^{\beta}}=1\right\} \\
=F / R,
\end{array}
$$

where $\alpha \geqq \beta>0$. Then $G$ has trivial multiplicator and has a presentation 


$$
G_{2}=\left\{a, b, c \mid c=a^{-1} b^{-1} a b, c^{-1} a c=a^{1-p^{\alpha}}, c b c^{-1}=b^{1+p^{\beta}}\right\} .
$$

Proof. Suppose we show $G_{1}$ has trivial multiplicator; then since it is shown in [2] that $G_{2}$ is a finite p-group the previous theorem gives the desired result. Hence we have proved the theorem when we have shown $G$ has trivial multiplicator. To do this it is sufficient to show that the first set of relations are a consequence of the second modulo $[F, R]$ or that $G_{1}$ has a presentation

$G_{3}=\left\{a, b, c \mid c=a^{-1} b^{-1} a b, c^{-1} a c=a_{1}^{-p^{\alpha}}, c b c^{-1}=b^{1+p^{\beta}}, a^{-1} c^{p^{\beta}} a=c^{p^{\beta}}, b^{-1} c^{p^{\beta}} b=c^{p^{\beta}}\right\}$ $=F / R$.

We have $c^{-p^{\beta}} a c^{p^{\beta}}=a$ whence the order of $a$ divides $\left(1-p^{\alpha}\right)^{p^{\beta}}-1$ and since we know $G$ is a finite $p$-group then $a^{p^{\alpha+\beta}}=1$. Similarly $b^{p^{2 \beta}}=1$. However with $a^{p^{x+\beta}}=b^{p^{2 \beta}}=1$ then equation (2.27) of [2], with $a$ and $b$ transposed, gives $c^{p^{\alpha}+p^{\beta}}=1$ whence $c^{\beta}=1$.

Similarly if $G$ is the finite $p$-group, $p \geqq 3$ with presentation

$G=\left\{a, b, c \mid c=a^{-1} b^{-1} a b, c^{-1} a c=a^{1-p^{\alpha}}, c b c^{-1}=b^{1-p^{\beta}}, a^{p^{\alpha+\beta}}=b^{p^{2 \beta}}=c^{p^{\beta}}=1\right\}$

where $\alpha>\beta>0$. Then $G$ has trivial multiplicator and has a presentation

$$
\left\{a, b, c \mid c=a^{-1} b^{-1} a b, c^{-1} a c=a^{1-p^{\alpha}}, c b c^{-1}=b^{1-p^{\beta}}\right\} .
$$

\section{Groups of order $3^{n}, n \leqq 6$}

The multiplicators for the groups of order $3^{n}, n \leqq 6$ were calculated by computer and it was found that there are eleven non abelian groups with trivial multiplicator, seven of which are metacyclic and have been considered in [6]. The four non metacyclic, non abelian groups with trivial multiplicator are, using the terminology of [1],

$$
\begin{aligned}
& G_{1}=\Delta_{6}(221) a=\left\{a, b, c \mid c=a^{-1} b^{-1} a b, c^{-1} a c=a^{-2}, c b c^{-1}=b^{4}, a^{9}=b^{9}=c^{3}=1\right\}, \\
& G_{2}=\Delta_{6}(321) a_{1}=\left\{a, b, c \mid c=a^{-1} b^{-1} a b, c^{-1} a c=a^{-8}, c b c^{-1}=b^{4}, a^{27}=b^{9}=c^{3}=1\right\}, \\
& G_{3}=\Delta_{6}(321) a_{2}=\left\{a, b, c \mid c=a^{-1} b^{-1} a b, c^{-1} a c=a^{-8}, c b c^{-1}=b^{-2}, a^{27}=b^{9}=c^{3}=1\right\},
\end{aligned}
$$

and

$$
\begin{gathered}
G_{4}=\Delta_{6}(221) c=\left\{a, b, c, d \mid c=a^{-1} b^{-1} a b, d=c^{-1} a^{-1} c a, c^{-1} b c=b^{-2},\right. \\
\left.a d=d a, b d=d b, a^{3}=b^{3}, b^{9}=c^{3}=d^{3}=1\right\} .
\end{gathered}
$$

$G_{1}, G_{2}$ and $G_{3}$ are special cases of theorem 2.5 so we need only consider $G_{4}$. Let $H$ be the group with presentation

$$
H=\left\{a, b, c, d \mid c=a^{-1} b^{-1} a b, d=c^{-1} a^{-1} c a, c^{-1} b c=b^{-2}, a^{3}=b^{3}\right\} .
$$

Since $b^{3}$ is central then we have immediately that $b^{9}=1$, and cubing the relation 
$c b^{-1}=a^{-1} b^{-1} a$ gives $c^{3}=1$. Suppose that $d$ is central then cubing $c d=a^{-1} c a$ gives that $d^{3}=1$, hence we have shown that $H=G_{3}$ when we have shown $d$ is central. Writing $[x, y]$ to denote $x^{-1} y^{-1} x y$ we have

whence

$$
\begin{aligned}
b^{-1} d b=b^{-1}[c, a] b & =\left[b^{-1} c b, b^{-1} a b\right]=\left[c b^{3}, a c\right]=b^{-3}[c, a c] b^{3}\left[b^{3}, a c\right] \\
& =[c, a c]=c^{-1}[c, a] c=c^{-1} d c
\end{aligned}
$$

$$
\left[c b^{-1}, d\right]=1 .
$$

Also we have that $a^{3}=b^{-1} a^{3} b=(a c)^{3}$, since $a^{3}$ is central, whence $a^{2}=c a c a c$ $=c a^{2} c d c=c a^{-1} c a^{3} d c=c^{2} d a^{2} d c$, giving that $a^{2} d c a^{-2}=d^{-1} c$ or $a^{-1} d c a$ $=d^{-1} c$. However $c^{3}=1$ implies $(c d)^{3}=1$ which implies $(d c)^{3}=1$ whence $a^{-1}(d c)^{2} a=\left(d^{-1} c\right)^{2}$ or $a^{-1} c^{-1} d^{-1} a=d^{-1} c d^{-1} c$ giving $d^{-1} c^{-1} c^{-1} d^{-1} a$ $=d^{-1} c d^{-1} c$ or $a^{-1} d^{-1} a=c^{-1} d^{-1} c$ yielding

$$
\left[c a^{-1}, d\right]=1 \text {. }
$$

Finally we have $a^{-1} b a=b c^{-1}=c^{-1} b^{4}$ whence $b a^{-1} b^{-1}=b^{-3} c a^{-1}$, giving on cubing that $a^{-3}=c a^{-1} c a^{-1}$, since $b^{3}=a^{3}$ is central, or $a^{-2}=c a^{-1} c a^{-1} c$ $=c^{2} d a^{-2} c$. Hence $c^{2} d a^{-2} c a=1$ or $c^{2} d a c a^{-1}=1$ and $d a c a^{-1} c^{-1}=1$, yielding that $d=c a c^{-1} a^{-1}=a c d c^{-1} a^{-1}$. This gives

$$
[a c, d]=1 \text {. }
$$

Whence (1), (2) and (3) give that $d$ is central and therefore $H=G_{4}$ giving a presentation for $G$ with an equal number of generators and relations. Note that the presentation $H$ may be modified to give a 2-generator, 2-relator presentation.

We have therefore shown that all groups of order $3^{n}, n \leqq 6$, with trivial Schur multiplicator have presentations with zero deficiency.

\section{References}

[1] Rodney K. James, The Groups of Order $p^{6}(p \geqq 3)$, Ph. D. Thesis University of Sydney, 1968.

[2] I. D. Macdonald, 'On a class of finitely presented groups', Canad.J. Math. 14 (1962), 602-613.

[3] B. H. Neumann, 'On some finite groups with trivial multiplicator', Publ. Math. Debrecen 4 (1955), 190-194.

[4] R. G. Swan, 'Minimal resolutions for finite groups', Topology 4 (1965), 193-208.

[5] J. W. Wamsley, 'Minimal presentations for certain group extensions', Israel J. Math. 9 (1971), 459-463.

[6] J. W. Wamsley, 'The deficiency of metacyclic groups', Proc. Amer. Math. Soc. 24 (1970), 724-726.

The Flinders University of South Australia 